ARTÍCULO ORIGINAL

Rev Colombiana Cienc Anim 2016; 8(Supl):293-296.

\title{
Melanoma ocular en un caballo criollo colombiano albino
}

\section{Ocular melanoma in a horse Colombian creole albino}

\author{
CARDONA A, JOSÉ ${ }^{*}$ Ph.D, BUITRAGO M, JHONNY² M.Sc, MONTES V, DONICER ${ }^{3}$ Ph.D.
}

\begin{abstract}
${ }^{1}$ Universidad de Córdoba, Departamento de Ciencias Pecuarias, Grupo de Investigaciones en Medicina de Grandes Animales (MEGA), Profesor Titular de Medicina y Clínica de Grandes Animales, Montería, Colombia. ${ }^{2}$ Universidad de Córdoba, Facultad de Medicina Veterinaria y Zootecnia, Estudiante de Maestría en Ciencias Veterinarias del Trópico, Grupo de Investigación en Medicina de Grandes Animales (MEGA), Montería, Colombia. ${ }^{3}$ Universidad de Sucre, Facultad de Ciencias Agropecuarias, Departamento de Zootecnia, Grupo de Investigación en Mejoramiento y Reproducción Animal, Sincelejo, Colombia.
\end{abstract}

\section{Keywords:}

Diagnostic; cancer; neoplasms; histopathology.

Palabras Clave:

Diagnóstico; cáncer; neoplasia; histopatología.
INFORMACIÓN

Recibido: 09-09-2016;

Aceptado: 18-11-2016.

Correspondencia autor:

cardonalvarez@hotmail.com

\section{Abstract}

The case of a Colombian Creole horse race, albino, neutered male, 10 years old, with the presence of hyperpigmented eye injury compatible with melanoma is exposed. Clinical evaluation was performed and pathological characterization of eye injury is hyperpigmented being located at the level of the sclera and upper eyelid of the left eye. Subsequently, after sedation and application of local anesthesia was taken an incisional biopsy of tissue from the periphery of the lesion located on the upper eyelid, then fixed in $10 \%$ formalin and taken to the Laboratory of Pathology Department of Animal Sciences at the University of Cordoba, Colombia, where they were processed to inclusion in paraffin. Finally, the sample was stained with hematoxylin - eosin (H-E), where the presence of large amounts of atypical melanocytes grouped in nests or tecas was ratified. The final diagnosis was ocular melanoma.

\section{Resumen}

Se expone el caso de un caballo de la raza Criollo Colombiano, albino, macho castrado, de 10 años de edad, con presencia de lesión ocular hiperpigmentada compatible con melanoma. Se le realizó evaluación clínica y caracterización anatomopatológica de la lesión ocular, siendo esta hiperpigmentada ubicada a nivel de la esclerótica y párpado superior del ojo izquierdo. Posteriormente, previa sedación y aplicación de anestesia local fue tomada una biopsia incisional de tejido desde la periferia de la lesión ubicada en el párpado superior, posteriormente fijada en formol al $10 \%$ y llevadas al laboratorio de Patología del Departamento de Ciencias Pecuarias de la Universidad de Córdoba, Colombia, donde fueron procesadas hasta su inclusión en parafina. Finalmente, la muestra fue teñida con la coloración de Hematoxilina - Eosina (H-E), donde se ratificó la presencia de grandes cantidades de melanocitos atípicos, agrupados en nidos o tecas. El diagnóstico definitivo fue de Melanoma ocular. 


\section{Introducción}

El melanoma, es una neoplasia originada por una alteración en el metabolismo de la melanina, lo que conlleva a la formación de nuevos melanoblastos, por acúmulo inadecuado de melanina en los melanófagos, posiblemente debido a una falla en la degradación de la melanina en la medida que el animal envejece y pierde pigmentación (LAUS et al. 2010).

Son varias las teorías sobre las posibles causas del Melanoma, pudiendo deberse a diversos factores, que van desde causas infecciosas (virales), inflamatorias, traumáticas, radiación ultravioleta (UV) hasta las causas genéticas, siendo identificado una duplicación de 4,6 kb en el intrón 6 del gen Syntaxin 17 (STX17) y la expresión del NR4A3 (Nuclear Receptor Subfamily 4, group A, member 3) como la mutación causante del fenotipo de caballos toldillos (grises), incluyendo el desarrollo del melanoma. Por lo que se sugiere que los melanocitos del folículo piloso y de la dermis tienen diferentes ciclos de vida (ROSENGREN et al. 2008). Sin embargo, SELTENHAMMER et al. (2004), indican el controversial papel que podría desempeñar la exposición prolongada a las radiaciones UV, postulandose la idea de que la hormona estimulante de $\alpha$ - melanocitos podría ser influenciado por las radiaciones solares, así las lesiones se ubiquen en regiones protegidas de la luz solar.

Los sitios considerados típicos de ubicación de melanomas son a nivel de la superficie ventral de la cola, región perianal y perineal, genitales (vulva, prepucio y pene), cuello, orejas, parpados y comisuras labiales (NAVARRO et al. 2012), mientras que los sitios de poca presentación considerados raros o atípicos, son a nivel ocular, bolsas guturales y esófago (MONTGOMERY, 2014).

Macroscópicamente, son masas tamaños variados y firmes, de apariencia nodular, infiltrativa o con apariencia de placas, pudiendo ser pigmentados o no, algunas veces ulcerados (SCHONIGER y SUMMERS, 2009).

Microscópicamente, en los Melanomas malignos se observa que el tamaño de las células y los núcleos difieren entre las células, y se observaron figuras mitóticas, siendo los tipos de células más comunes las epitelioides pleomórficas y redondas, las áreas tumorales son difusamente definidas, y la pigmentación puede ir desde muy pigmentada a zonas amelanóticas (MOUSSA et al. 2011). Entre los tratamientos, indicados para el Melanoma se encuentran el uso de quimioterapia, electroquimioterapia con cisplatina intratumoral y cimetidina vía oral, de igual forma está recomendada la criocirugía y la remoción quirúrgica (LAUS et al. 2010; SPUGNINI et al. 2011). El objetivo de este trabajo fue realizar un informe sobre los aspectos clínicos e histopatológicos de un caso del melanoma ocular en un caballo albino criollo colombiano en el departamento de Córdoba, Colombia.

\section{Materiales y métodos}

El semoviente en estudio, fue un equino de la raza Criollo Colombiano, macho castrado, color blanco albino, de 10 años de edad, $380 \mathrm{~kg}$ de peso aproximadamente, procedente del municipio de Montelibano (Córdoba, Colombia), fue atendido por el servicio clínico ambulatorio de grandes animales de la Facultad de Medicina Veterinaria y Zootecnia de la Universidad de Córdoba, Colombia. Fue realizado examen clínico general y ocular especializado, consistente en evaluación de la región ocular izquierda, la cual presentó lesión pigmentaria unilateral a nivel de esclerótica y con apariencia tumoral exofítica en el parpado superior (Figura 1) compatibles con Melanoma ocular.

Posteriormente, previa sedación (Xilacina 10\%, $\mathrm{Erma}^{\circledR}$, Colombia) y aplicación de anestesia local (Lidocaina $2 \%$, Synthesis ${ }^{\circledR}$, Colombia) fue tomada una biopsia incisional de tejido desde la periferia de la lesión ubicada en el párpado superior izquierdo, posteriormente fijada en formol al $10 \%$ y llevadas al laboratorio de Patología del Departamento de Ciencias Pecuarias de la Universidad de Córdoba, Colombia, donde fueron procesadas hasta su inclusión en parafina. La muestra fue teñida con coloración de Hematoxilina - Eosina (H-E), para ratificar la presencia de melanocitos atípicos, agrupados (en nidos o tecas). 


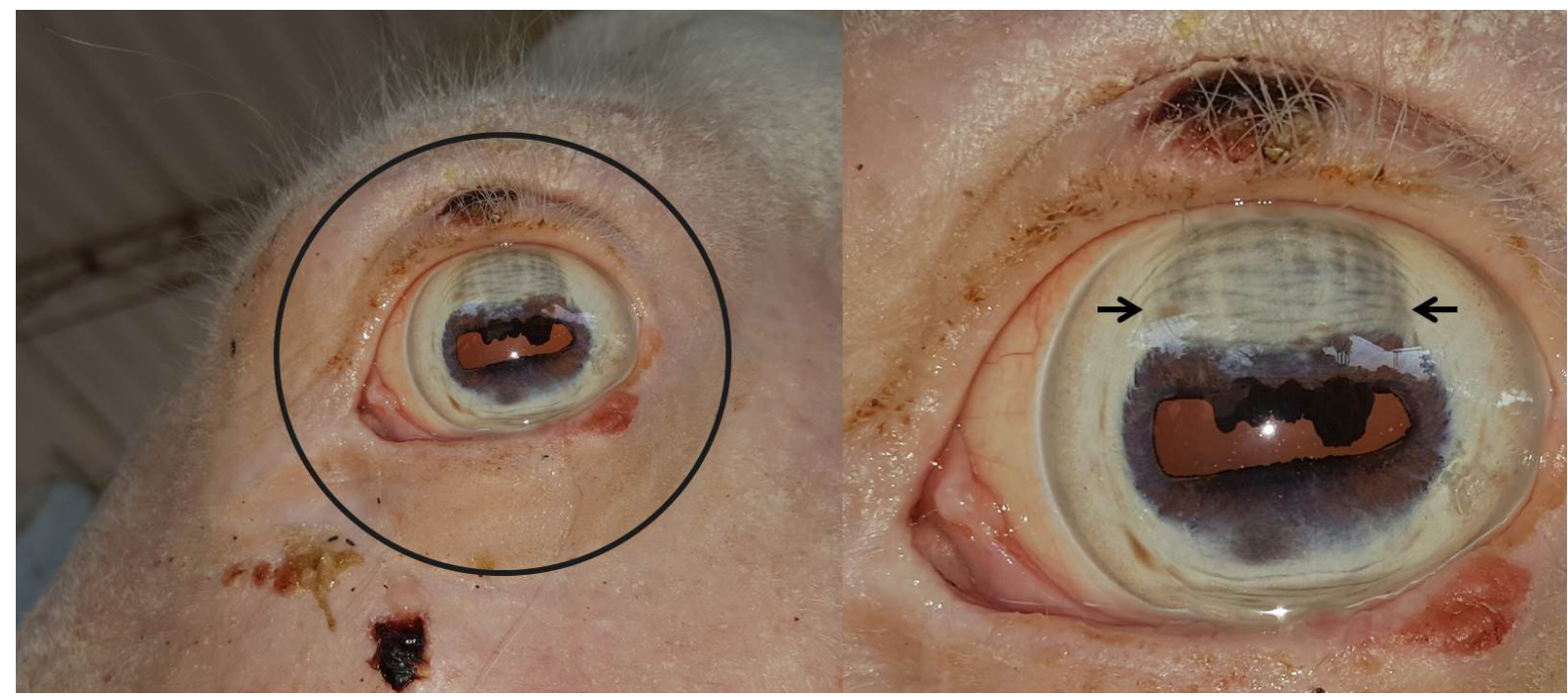

Figura 1. Lesión pigmentaria unilateral izquierda a nivel de esclerótica y de apariencia tumoral exofítica en el parpado superior (círculo y flecha).

\section{Resultados y Discusión.}

Las características anatomopatológicas de las lesiones obtenidas en el examen clínico, así como las características histopatológicas de la biopsia teñida con HE (Figura 2) confirmaron el diagnóstico de Melanoma ocular en un caballo blanco albino estudiado de acuerdo con lo reportado como método diagnóstico del Melanoma equino (SCHONIGER y SUMMERS, 2009; LAUS et al. 2010; MOUSSA et al. 2011; NAVARRO et al. 2012). El Melanoma, es considerado una de las neoplasias dermatológicas más frecuentes en equinos, pudiendo llevar a complicaciones metastásicas especialmente en caballos toldillos (LAUS et al. 2010), siendo la segunda neoplasia dermatológica de los caballos, después del sarcoide o del carcinoma de células escamosas, representando del 5 al $14 \%$ de las neoplasias cutáneas (SPUGNINI et al. 2011).

El melanoma evaluado, presento localización ocular, siendo esta, una ubicación atípica y contraria a los reportado por NAVARRO et

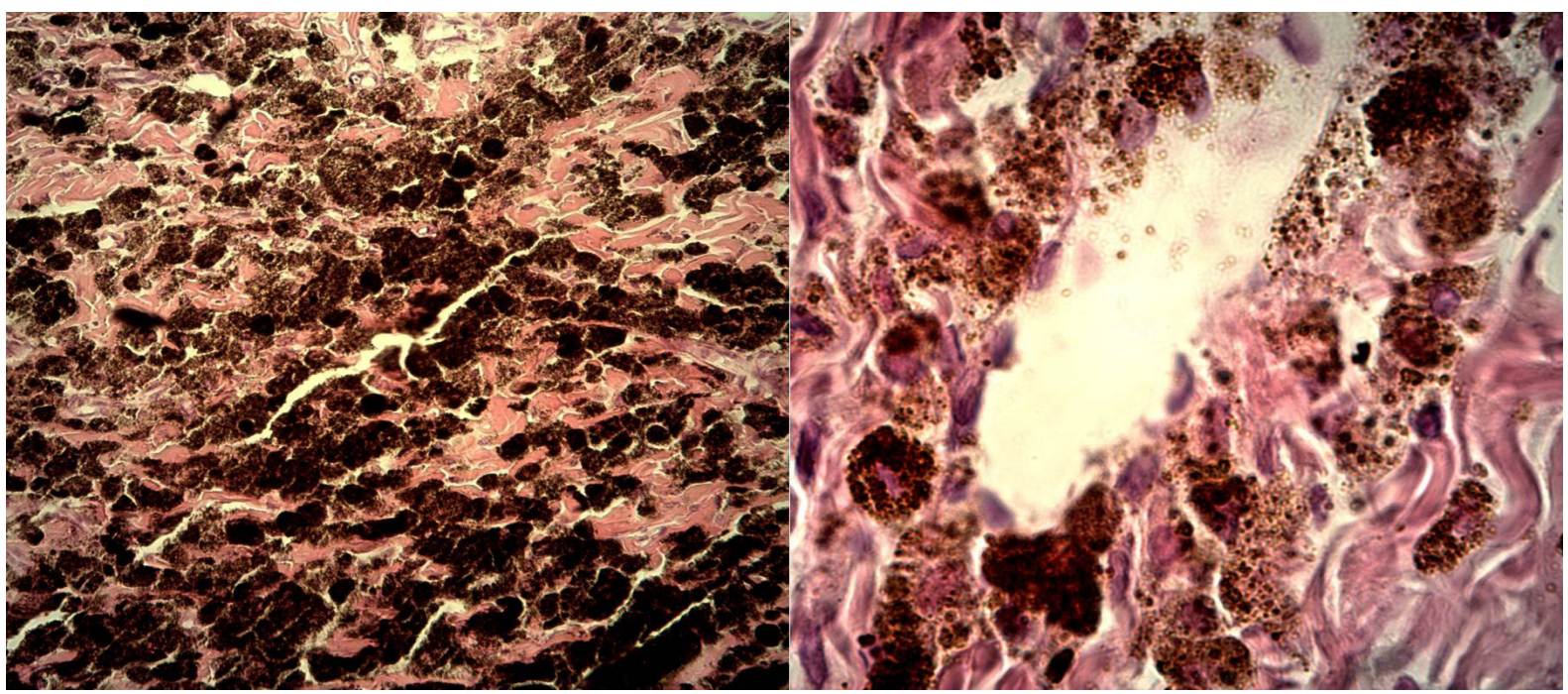

Figura 2. Tinción de H-E, se puede observar la presencia de grandes cantidades de melanocitos atípicos, agrupados (en nidos o tecas). 10X y $40 \mathrm{X}$ respectivamente. 
al. (2012) y PHILLIPS y LEMBCKE (2013), quienes manifestaron, que ese tipo de lesión, principalmente se presentan en áreas como región perineal, anal, perianal y genital (vulva, pene y prepucio) y en menor proporción en regiones como cuello, comisura labial y mucosa oral.

Los hallazgos histopatológicos del presente estudio con la coloración de H-E (Figura 2), se corresponden con los reportados en la literatura para el diagnóstico del Melanoma (LAUS et al. 2010; MOUSSA et al. 2011; SPUGNINI et al. 2011; PHILLIPS y LEMBCKE, 2013), confirmado por la presencia de células epitelioides pleomórficas y redondas de tamaño variable, así mismo, las áreas tumorales eran difusamente definidas, fuertemente pigmentadas y marcada presencia de melanocitos atípicos agrupados (en nidos o tecas). Por la ubicación geográfica del caso (departamento de Córdoba, Colombia), se cree que caballos moros o tordillos que viven en países de clima tropical con alta incidencia de luz ultravioleta (UV) están más predispuestos, debido a que se sospecha que el aumento de la producción de a-MSH (hormona estimulante de $\alpha$ - melanocitos), influenciada por la luz solar, pueda estar envuelto en la patogénesis de estas neoplasias (SELTENHAMMER et al. 2004; ROSENGREN et al. 2008).

\section{Referencias}

LAUS F, CERQUETELLA M, PAGGI E, IPPEDICO G, ARGENTIERI M, CASTELLANO G, SPATERNA A, TESEI B. 2010. Evaluation of cimetidine as a therapy for dermal melanomatosis in grey horse. Israel J. Vet. Med. 65(2): 48-52.

MONTGOMERY K. 2014. Equine ocular neoplasia: A review. Equine Vet. Educ. 26 (7):372-380.

MOUSSA R, SEVASTRE B, TAULESCU M, BOLFĂ P, GAL A, TABARAN F. 2011. Histological and Macroscopical Study of Horse Melanoma. Bulletin UASVM, Vet. Med. 68 (1): 252-259.

NAVARRO C, RODRÍGUEZ M, FUENTES E. 2012. Melanoma maligno palpebral en un equino: reporte de un caso. Rev. Sist. Prod. Agroecol. 3(1): $139-145$.

PHILLIPS J, LEMBCKE L. 2013. Equine Melanocytic Tumors. Vet Clin Equine. 29 (3):673-687.

ROSENGREN G, GOLOVKO A, SUNDSTRÖM E, CURIK I, LENNARTSSON J, SELTENHAMMER M. 2008. A cis-acting regulatorymutation causes premature hair graying and susceptibility to melanoma in the horse. Nat Genet. 40: 1004-1009.

SCHONIGER S, SUMMERS B. 2009. Equine skin tumours in 20 horses resembling three variants of human melanocytic naevi. Vet. Dermatol. 20 (3): 165-173.

SELTENHAMMER M, HEERE-RESS E, BRANDT S, DRUML T, JANSEN B, PEHAMBERGER H, NIEBAUER G. 2004. Comparative histopathology of Grey horse melanoma and human malignant melanoma. Pigment. Cell. Res. 17(6): 674-681.

SPUGNINI E, D'ALTERIO G, DOTSINSKY I, MUDROV T, DRAGONETTI E, MURACE R, CITRO G, BALDI A. 2011. Electrochemotherapy for the Treatment of Multiple Melanomas in a Horse. J. Equine Vet. Sci. 31 (8): 430-433. 